\title{
Analysis on the electrical conductivity of microstructure of the solar panels power supply system of gelatinization equipment
}

\author{
Minghui $\mathrm{You}^{1}$, Xinyu $\mathrm{Yu}^{2}$, Qixiang Sun ${ }^{1}$, Liping $\mathrm{Yin}^{1}$, Shijun $\mathrm{LI}^{1}$, \\ Xue $\mathrm{Li}^{1}$, Jingsheng $\mathrm{Liu}^{1, \text { a }}$ \\ ${ }^{1}$ Jilin Agricultural University, Changchun 130118, Jilin, China \\ ${ }^{2}$ Jilin Institute of agricultural machinery, Changchun 130021, Jilin, China \\ amhyou000@163.com
}

Keywords: power electronics; semiconductor switches; the V-A Characteristics

\begin{abstract}
Diode is the foundation of semiconductor devices.This paper analysis the electrical conductivity of microstructure of solar panels in the Power supply system of gelatinization equipment. We discussed the formation process of PN junction, theoretical analysis of electrical conductivity.In this paper we analyze linear region of voltage - current characteristic. From the microscopic point of view, it analyzes the static structure and dynamic change, and provides basic data for its application.
\end{abstract}

\section{Introduction}

The rapid development of modern electronic technology, for people's culture, material life provides a superior condition, digital camera, air conditioning, electronic computer, etc[1], were typical examples of the application of electronic technology. [2]The diode was the foundation of the semiconductor device, and the specific manufacturing process is adopted. In a pure semiconductor side doped with pentavalent elements, made of $\mathrm{N}$ type semiconductor; on the other side of the incorporation of trivalent elements made of $\mathrm{P}$ type semiconductor, at their junctions formed a p-n junction. [3]In practical applications, a p-n junction diode can be formed, the two PN structure of a transistor, a plurality of PN junctions constitute the integrated circuit. [4] To add a PN junction forward voltage, forming the diffusion current is larger, the PN junction was conducted. For PN junction with reverse voltage, because the number of minority carriers was very few, so the reverse current was very small. So the PN junction in the off state. Due to the component itself, the volt ampere characteristics can judge its performance, so the paper focuses on the analysis of the characteristics of the diode's volt ampere characteristics. [5]

\section{The formation of PN junction}

The hole was positively charged and negatively charged electrons free. [6]Electron and hole could be free, they would form the current directional movement, therefore refer to them as the carrier. In the $\mathrm{P}$ region, the majority of the carriers were holes, minority carriers were electrons in the.N region.They got an electronic trivalent impurity ions, negatively charged,and lost an electron pentavalent impurity ions, positively charged.These ions were bound to the covalent bond in the crystal, were not free to move. Matter was always moving from high concentration to low concentration, which was the result of concentration difference, which was called diffusion. This was the first diffusion occurred in both sides of the interface. After an immeasurably short time interval,the electron - positron pairrecombined. This left a number of negative ions in the $\mathrm{P}$ area and some positive ions in the $\mathrm{N}$ area near the interface.Both sides of the $\mathrm{P}$ type semiconductor and $\mathrm{N}$ type semiconductor interface on the formation of a space charge region. The space charge region was a p-n junction. Because of free electrons and holes in semiconductor the concentration difference between the diffusion movement. With the diffusion movement, in the two side of the interface to form a space charge in this area, the space charge region was a p-n junction. 


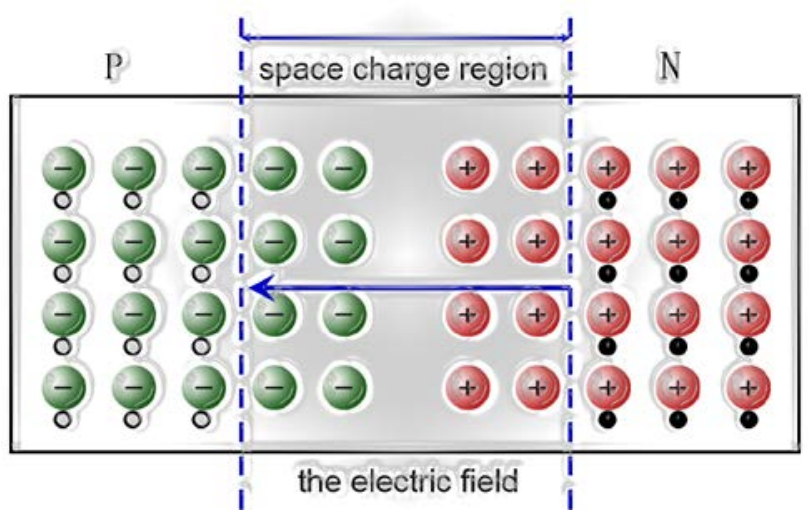

(a)

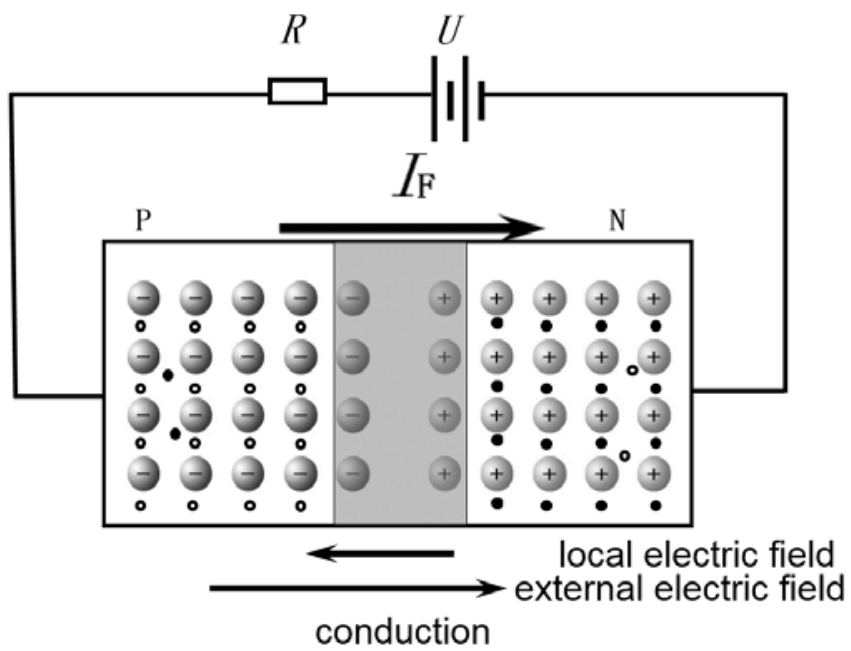

(b)

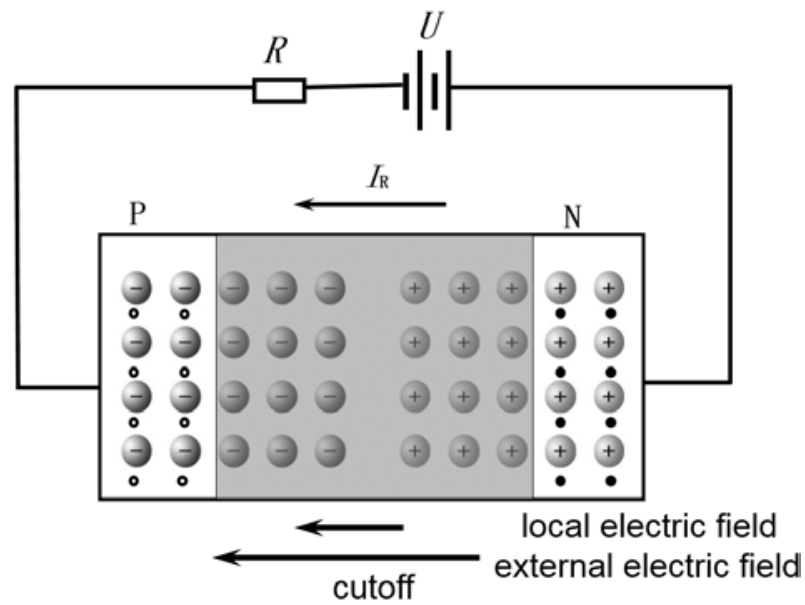

(c)

Fig. 1 The formation of PN junction(a)(b)(c)

The movement of diffusion and drift motion were both interrelated and contradictory. [7]In the ea rly stage of forming space charge region, As the space charge region was not wide enough, the majo rity of the majority of the carrier's diffusion was dominant. However, with the diffusion motion, the space charge region was gradually widened, and the electric field was gradually enhanced. The diffu sion motion of majority carriers was gradually weakened, and the d7ift motion of minority carriers was gradually enhanced. Finally, the diffusive motion and the drift motion reached a dynamic equili brium. P area and $\mathrm{N}$ area were mutual diffusion and drift to the other side of the same number of car riers. After reaching the dynamic equilibrium, the width of the space charge region was basically fix 
ed, and was in a relatively stable state. PN junction formed completely. In practical applications, the two PN structure of a transistor, a plurality of PN junctions constituted the integrated circuit.

\section{Voltage - current characteristic}

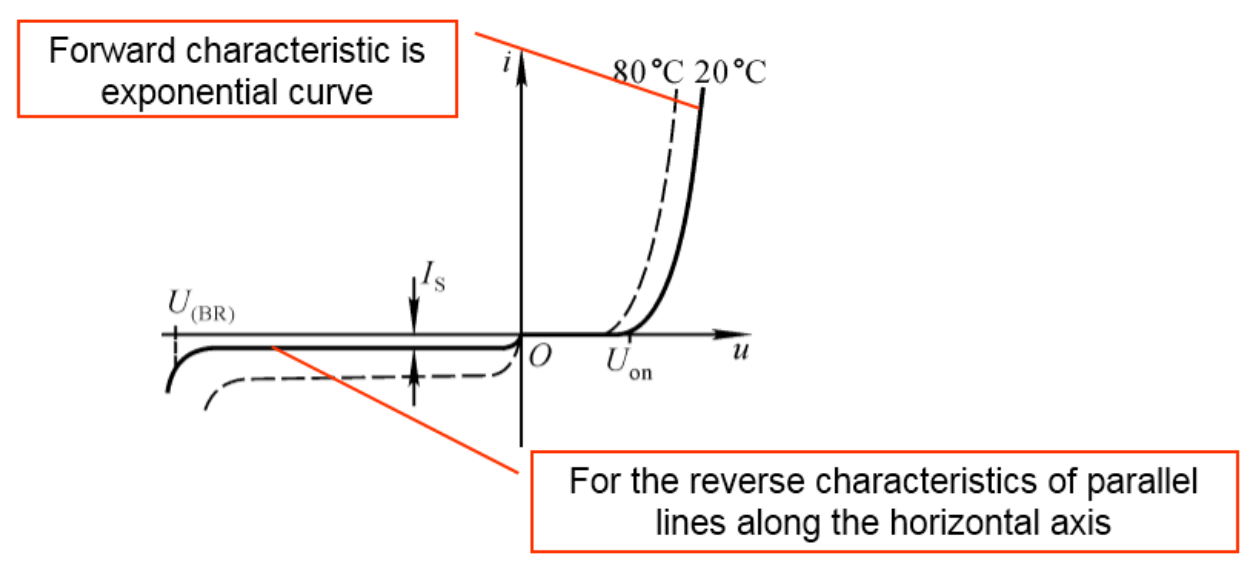

Fig. 2 Voltage - current characteristic

We can see from the figure, when the applied voltage was very small, the current was very small, almost zero, this was because when the applied voltage was very small, the external electric field was not strong enough, not enough to overcome the internal field motion of the majority carrier diffusion resistance. Therefore, the forward current was almost zero. But when the forward voltage exceeds a certain value, internal electric field was weakened, the current increased rapidly. When the applied reverse voltage to the PN junction, in a large range of voltage, current was very small, almost zero, and when the reverse voltage increases to a certain value, the reverse current increased rapidly, the PN junction lost one-way conductivity, this phenomenon was called reverse breakdown.

\section{Summary}

This paper analyzes the formation and characteristics of PN junction. The space charge region was a p-n junction. Because of free electrons and holes in semiconductor the concentration difference between the diffusion movement. With the diffusion movement, in the two side of the interface to form a space charge in this area, the space charge region was a p-n junction.The unidirectional conductivity has very important application in practice.

\section{Acknowledgments}

The authors wish to express their gratitude to the projects: Jilin Province Education Department Project (No. 2015Y174), Jilin Agricultural University learning program, Jilin Provincial Department of human resources and social security Project (No. 2015Y24)for their generous support of this work.

\section{References}

[1]Chen Da,The Study of 3C-SiC/Si Heteroepitaxial Growth and I-V characteristics of Schottky diodes[D], Xidian University,2011,50-56.

[2]Lian Hanli, Theory analysis on volt-ammetry characteristic curves of diode [J] Journal of Xi'An University of Post and Telecommunication, 2008.13(5):150-152.

[3]Wang Wenting,Diode Volt-ampere Characteristic Automatic Test System. [J]Instrument Technique and Sensor,2015.11:65-66. 
[4]Liu Jiaju, Oscilloscope measurement diode volt-ampere characteristics. [J] Journal of Chifeng University(Natural Science Edition),2014.30(1):1-2.

[5]Liu Chaoying, Understanding the Characteristics of Semiconductor Switches by the V-A Characteristics . [J] Journal of Zhao Qing University,2016,37(5):28-32.

[6]Sun Chengzheng,Analysis of the characteristics of photovoltaic cells. [J] Journal of Jing Chu University of Technology,2016,31(4):25-29.

[7]Xu Sheng ,Study on the new models of high power LED current-voltage characteristics. [J] Journal of Optoelectronics Laser,2016,26(11):2076-2082. 